\title{
Associations between antiretroviral therapy-related experiences and mental health status among people living with HIV in China: A prospective observational cohort study
}

Jinzhao Xie

Zixin Wang

Quanmin Li

Qiangsheng He

Guohong Xu

Yonghong Li

Kai Zhou

Linghua Li

Jing Gu ( $\sim$ gujing5@mail.sysu.edu.cn )

https://orcid.org/0000-0001-5436-2339

\section{Research}

Keywords: HIV, Antiretroviral therapy, Mental health, China

Posted Date: January 28th, 2021

DOI: https://doi.org/10.21203/rs.3.rs-80094/v2

License: (c) This work is licensed under a Creative Commons Attribution 4.0 International License. Read Full License 


\section{Abstract}

Background: Mental health problems (e.g., depression and anxiety) are among the most commonly reported comorbidities of HIV. Antiretroviral therapy (ART) coverage has increased sharply. The purposes of this prospective cohort study were to investigate the ART-related experiences and whether they were associated with mental health problems among a sample of people living with HIV undergoing ART in China.

Methods: The participants were 400 people living with HIV who had started ART for the first time in Guangzhou city. They were followed-up one-year after ART initiation. Probable depression and moderate/severe anxiety were measured at baseline and Month 12, while experiences related to ART (e.g., side effects and regained selfconfidence) were measured at Month 6 . Univariate and multivariate logistic regressions were used to explore the associations between baseline characteristics, ART-related experiences and mental health status.

Results: Among the 300 participants (75.0\%) who completed all three surveys, a significant decline in prevalence of probable depression (23.0\% at baseline vs. $14.0 \%$ at Month $12, P=0.002)$ and moderate/severe anxiety (14.7\% at baseline vs. $8.7 \%$ at Month $12, P=0.023$ ) was observed during the follow-up period. After adjustment for mental health status and potential confounders at baseline, a number of ART-related experiences at Month 6 were associated with probable depression and/or moderate/severe anxiety measured at Month 12 . Improved physical health, relationships with sexual partners, and self-confidence were associated with decreased mental health issues, while the side effects of ART, AIDS-related symptoms, and inconvenience in daily life due to ART use were associated with increased mental health issues.

Conclusions: ART-related experiences were associated with mental health problems, tailored mental health promotion interventions targeting these experiences are needed.

\section{Background}

Antiretroviral therapy (ART) has increased the life expectancy of people living with HIV (PLWH), allowing them to live longer with this chronic medical condition [1, 2]. In line with the World Health Organization (WHO), China renewed its national ART guidelines in 2016 [3], and ART coverage increased from 67\% in 2015 to $80 \%$ in 2017 [4]. After entering the "treat all" era [5], ART coverage and effectiveness has increased sharply across countries $[6,7]$. Recently, researchers have shifted their focus toward the next targets following viral suppression. The UNAIDS added a fourth 90 target (i.e., ensuring that $90 \%$ of people with viral load suppression have good healthrelated quality of life) to the existing agenda [8].

Mental health problems (e.g., depression and anxiety) are among the most commonly reported comorbidities of HIV [9-11], affecting quality of life and wellbeing among PLWH $[12,13]$. Evidence suggests that depression and anxiety are associated with low medical adherence [14], worse retention in HIV care [15, 16], and poor HIV-related outcomes (e.g., quality of life) $[17,18]$. A meta-analysis has shown an overall prevalence of depression and anxiety of $33.6 \%$ and $28.4 \%$, respectively, among PLWH on ART [19]. In China, the prevalence of depression was reported as about $40 \%$ among PLWH on ART $[20,21]$. Given these findings, the prevention and treatment of mental health problems may be an important element of HIV treatment and care.

A growing body of literature has demonstrated improved mental health status among PLWH after ART initiation. A longitudinal study in the United States showed that, compared to pre-treatment levels, the prevalence of 
depression decreased from $30 \%$ to $21 \%$ after one year on ART [22]. It is possible that the benefits of ART are mediated via a reduction in inflammatory pathways that affect the risk of depression, such as the kynurenine pathway or tryptophan catabolism [23]. On the other hand, ART may also indirectly improve mental health status among PLWH by increasing access to health care services, psychological services and community support [24]. Although previous studies have identified a number of factors that are associated with mental health among PLWH on ART (e.g. gender [25], personal income level [26], living arrangements [27], and family support [21]), most of these studies were cross-sectional and ignored therapy-related experiences.

ART requires lifelong treatment, which brings both positive and negative experiences for users. On the one hand, ART brings significant clinical benefits for PLWH, such as improving physical health status, facilitating immune system recovery (e.g., higher CD4 cell counts), and slowing disease progression [28, 29]. There is also evidence that viral suppression by ART greatly reduces the risk of transmitting HIV to sexual partners [1, 2], potentially improving relationships between PLWH and their sexual partners. On the other hand, although advances in ART have largely reduced its side effects, recent studies have shown that about 25.0-53.3\% of PLWH still experience severe side effects in their first year after ART initiation [30,31]. Moreover, the daily regimen of ART is inconvenient for PLWH. For example, $25 \%$ of PLWH in eight high-income countries agreed that being tied to daily medication limited their day-to-day life, and $29 \%$ felt stressed by the need to take their medication at the right time every day [32]. These experiences related to ART may also influence mental health among PLWH. Previous studies found that the side effects of ART, lowered CD4 cell counts, and AIDS-related symptoms after ART initiation were associated with depression and anxiety [20,26,27]. However, these studies were limited by their cross-sectional nature and the lack of inclusion of potentially relevant experiences of ART.

As far as we know, no longitudinal study has investigated ART-related experiences and whether they were associated with mental health problems among PLWH. To address these gaps, this prospective cohort study measured ART-related experiences at Month 6 and mental health problems at Month 12 among a sample of PLWH in China who had initiated ART for the first time, and investigated whether ART-related experiences (Month 6) were associated with mental health problems (Month 12) after considering potential confounders.

\section{Methods}

\section{Study population and procedure}

This prospective cohort study was conducted in Guangzhou, China, from June 2016 to May 2018. Face-to-face interviews were conducted at baseline (the first day of ART admission), and at Month 6 and Month 12 after ART admission. The inclusion criteria were that the subjects were 1) aged 18 years or above, 2) HIV seropositive, 3) initiating ART for the first time, and 4) willing to attend surveys at baseline, Month 6, and Month 12. Those who had major psychiatric illnesses (schizophrenia or bipolar disorder) or could not communicate with the interviewers were excluded.

The participants were recruited from the ART clinic of Guangzhou Eighth People's Hospital in Guangzhou. The Guangzhou Eighth People's Hospital was the first ART centre in Guangdong province and has provided ART to over 10,000 PLWH since 2004 [33]. During the recruitment period (June 2016 to May 2017), trained nurses screened the new ART users for eligibility and referred those who met the criteria to the research team. The trained interviewers then reconfirmed the participants' eligibility, explained the details of the study, and assured 
them that refusal would not affect their right to use any related services and that they could quit the study at any time without being questioned. After providing written informed consent, the participants completed a selfadministered questionnaire which took about 15 minutes. They were invited to visit the hospital again to complete two other self-administered questionnaires after six and twelve months. On completion of each of the three surveys, the participants received a souvenir (worth 15 RMB or 2 USD) in appreciation of their time. Ethical approval for the study was obtained from the Institutional Review Board (IRB) of the School of Public Health, Sun Yat-sen University, Guangzhou, China (No: 2016-003).

Of the 480 prospective participants approached, 425 passed the eligibility screening. Of these, 400 provided written informed consent and completed the baseline survey (response rate: 94.1\%, 400/425), among whom 353 $(88.3 \%, 353 / 400)$ completed the Month 6 follow-up survey, and $300(75.0 \%, 300 / 400)$ also completed the Month 12 survey. Of the participants who did not complete both follow-up surveys $(n=100), 12$ were lost to clinic followup (didn't come back since the last visit), 11 were transferred to other ART clinics, 2 were died, 4 refused to participate in the follow-up surveys, and 71 didn't show up during the appointed follow-up period but remained in the treatment.

\section{Measurements}

\section{Mental health problems measured at baseline and Month 12}

Depressive symptoms were measured by the validated Chinese version of the Patient Health Questionnaire (PHQ-9) $[34,35]$. The PHQ-9 has been widely used to screen for depression in different Chinese populations [34, 35], including PLWH [36], and has acceptable reliability and validity. The scale contains nine items regarding the frequency of certain negative feelings over the past two weeks (response categories: $0=$ never, $1=$ sometimes, 2 $=$ often, $3=$ almost every day). The total scores ranged from 0 to 27 , and a higher score indicated more severe depression. A score of $\geq 10$ was used to define probable depression $[22,26]$.

Generalized anxiety disorder was measured by the validated Chinese version of the Generalized Anxiety Disorder Scale (GAD-7), which has shown acceptable reliability and validity in prior research [37]. The scale contains 7 items, with a score range of $0-21$, a higher score representing greater anxiety. In this study, a score of $\geq 10$ was used to define moderate/severe anxiety [38].

\section{Experiences related to ART measured at Month 6}

A panel of HIV epidemiologists, behavioural health researchers, nurses, and physicians from the ART clinic was formed to develop the questions regarding patients' experiences related to ART. The questions were then pilot tested among 15 PLWH who were currently on ART. Based on their feedback, the experience questions were revised to form the experiences measurement, which included four positive and four negative experiences.

Four items measured positive experiences related to ART. The participants were asked to report whether they had had the following experiences during the past six months: 1) a rise in CD4 cell count, 2) improvement in physical health, 3) improvement in relationships with sexual partners, and 4) regained self-confidence (response categories: 1 = yes, $0=$ no). Negative experiences related to ART in the past six months were also measured by four items: 1) side effects of ART, 2) AIDS-related symptoms, 3) inconvenience of ART use in daily life, and 4) exposure of HIV-positive status due to ART use (response categories: 1 = yes, 0 = no). 


\section{Baseline characteristics}

The participants were asked about their background characteristics, including age, sex, education, current marital status, monthly personal income, employment status, and city of permanent residence. HIV/AIDS-related characteristics were also recorded, including AIDS-related symptoms, CD4 cell count at the most recent test, route of HIV transmission (sexual behaviour with same-sex partner, sexual behaviour with opposite-sex partner, intravenous drug use, mother-to-child transmission, blood transfusion, occupational exposure, or not sure), and ART regime.

Psychosocial status including social support and social stigma was also measured at baseline. Social support was measured by the validated Chinese version of the Multidimensional Scale of Perceived Social Support (MSPSS) [39, 40]. The MSPSS contains 12 items and 3 dimensions including family, friends, and significant others. The score range for each dimension is $1-7$, with a higher score indicating better support. We used the social stigma module of the Chinese Courtesy Stigma Scales (CSSSs) to measure the social stigma patients experienced [41]. The scale contains 13 items, with a score range of 13-52, a higher score representing greater stigma.

\section{Statistical Analysis}

The baseline characteristics of those who completed all follow-up surveys and those who did not were compared using a chi-square test (for categorical variables), independent samples t-test (for continuous variables with normal distribution), or non-parametric test (for ranked variables and continuous with nonnormal/skewed distribution). Further analysis was performed among those who completed all three surveys: baseline, Month 6, and Month $12(n=300)$.

The depression and anxiety status were presented using both scores of assessments (continuous variables) and clinically defined thresholds (categorical variables). The difference in mental health problems between baseline and Month 12 was tested by the Wilcoxon signed rank test (for scores) and the paired-sample Chisquare test (for categorical mental health statuses). Associations between baseline characteristics and probable depression and moderate/severe anxiety measured at Month 12 were described by the odds ratio (OR) obtained from univariate logistic regression models. Multivariate stepwise logistic regression models were used to identify baseline factors that independently affected mental health status at Month 12 . To test whether ARTrelated experiences measured at Month 6 were associated with future mental health problems (probable depression and moderate/severe anxiety measured at Month 12), both univariate and adjusted logistic regression models were applied, adjusted for baseline variables with $P<0.1$, plus baseline mental health status. R software version 4.0.2 was used for the data analysis, and $P<0.05$ was considered statistically significant.

\section{Results}

\section{Baseline characteristics of participants}

Of the 400 participants, most were male (92.2\%), more than 25 years old $(79.6 \%)$, currently single $(55.8 \%)$, and non-permanent residents of Guangzhou (77.7\%). About $60 \%$ of the participants reported that they became infected through sexual behaviour with a same-sex partner. Over $80 \%$ used a combination of tenofovir, lamivudine, and efavirenz (84.0\%) as their ART regimen. At baseline, the median and lower and upper quartiles 
$\left[\mathrm{M}\left(\mathrm{Q}_{1}, \mathrm{Q}_{2}\right)\right]$ of depressive and anxious scores were $7.00(4.00,10.00)$ and $6.00(2.00,7.00)$ respectively, the prevalence of probable depression and moderate/severe anxiety was $26.0 \%$ and $15.0 \%$, respectively.

Those who did not complete both follow-up surveys were more likely to be older, have a lower or no fixed monthly personal income, have probable depression at baseline, and not have been infected through sexual behaviour with a same-sex partner (Table 1).

Baseline characteristics of all participants and by follow up status 


\begin{tabular}{|c|c|c|c|c|}
\hline \multirow[t]{2}{*}{ Variables } & $\begin{array}{c}\text { All } \\
\text { participants } \\
(n=400)\end{array}$ & $\begin{array}{c}\text { Participants who } \\
\text { completed follow- } \\
\text { up surveys } \\
(n=300)\end{array}$ & $\begin{array}{c}\text { Participants who did } \\
\text { not complete follow- } \\
\text { up surveys } \\
(n=100)\end{array}$ & $P$ \\
\hline & $n(\%)$ & $n(\%)$ & $n(\%)$ & \\
\hline $\begin{array}{l}\text { Background characteristics } \\
\text { Age (years) ( ) }\end{array}$ & $\begin{array}{c}32.62 \pm \\
9.72\end{array}$ & $31.88 \pm 9.03$ & $34.78 \pm 11.33$ & 0.010 \\
\hline $18-$ & $82(20.4)$ & $65(21.7)$ & $17(17.0)$ & 0.020 \\
\hline $25-$ & $171(42.8)$ & $133(44.3)$ & $38(38.0)$ & \\
\hline 35- & $92(23.0)$ & $70(23.3)$ & $22(22.0)$ & \\
\hline 45- & $55(13.8)$ & $32(10.7)$ & $23(23.0)$ & \\
\hline Sex & & & & 0.105 \\
\hline Female & $31(7.8)$ & $19(6.3)$ & $12(12.0)$ & \\
\hline Male & $369(92.2)$ & $281(93.7)$ & $88(88.0)$ & \\
\hline Education & & & & 0.082 \\
\hline Primary school and below & $133(33.2)$ & $91(30.3)$ & $42(42.0)$ & \\
\hline Junior or senior high & $102(25.6)$ & $82(27.4)$ & $20(20.0)$ & \\
\hline College and above & $165(41.2)$ & $127(42.3)$ & $38(38.0)$ & \\
\hline Current marital status & & & & 0.757 \\
\hline $\begin{array}{l}\text { Married or cohabiting with a } \\
\text { partner }\end{array}$ & $136(34.0)$ & $104(34.7)$ & $32(32.0)$ & \\
\hline Single & $223(55.8)$ & $167(55.6)$ & $56(56.0)$ & \\
\hline Other & $41(10.2)$ & $29(9.7)$ & $12(12.0)$ & \\
\hline $\begin{array}{l}\text { Monthly personal income in RMB } \\
\text { (USD) }\end{array}$ & & & & 0.005 \\
\hline No fixed income & $79(19.8)$ & $50(16.7)$ & $29(29.0)$ & \\
\hline$<3000(<433$ USD $)$ & $97(24.2)$ & $70(23.3)$ & $27(27.0)$ & \\
\hline 3000- (433- USD) & $127(31.8)$ & $108(36.0)$ & $19(19.0)$ & \\
\hline 5000- (722- USD) & $97(24.2)$ & $72(24.0)$ & $25(25.0)$ & \\
\hline Employment status & & & & 0.814 \\
\hline Full-time & $238(59.5)$ & $177(59.0)$ & $61(61.0)$ & \\
\hline $\begin{array}{l}\text { Part- } \\
\text { time/unemployed/retired/student }\end{array}$ & $162(40.5)$ & $123(41.0)$ & 39 (39.0) & \\
\hline City of permanent residence & & & & 0.627 \\
\hline Guangzhou & $89(22.3)$ & $69(23.0)$ & $20(20.0)$ & \\
\hline Other cities & $311(77.7)$ & $231(77.0)$ & $80(80.0)$ & \\
\hline \multicolumn{4}{|l|}{ HIV/AIDS-related characteristics } & \\
\hline $\begin{array}{l}\text { Presence of AIDS-related } \\
\text { symptoms }\end{array}$ & & & & 0.343 \\
\hline No & $246(61.5)$ & $189(63.0)$ & $57(57.0)$ & \\
\hline Yes & $154(38.5)$ & $111(37.0)$ & $43(43.0)$ & \\
\hline CD4 cell count (cell $\left./ \mathrm{mm}^{3}\right)$ & & & & 0.304 \\
\hline$<200$ & $112(28.0)$ & $78(26.0)$ & $34(34.0)$ & \\
\hline $200-$ & $173(43.2)$ & $135(45.0)$ & $38(38.0)$ & \\
\hline $350-$ & $75(18.8)$ & 59 (19.7) & $16(16.0)$ & \\
\hline $500-$ & $40(10.0)$ & $28(9.3)$ & $12(12.0)$ & \\
\hline Route of HIV transmission & & & & 0.013 \\
\hline $\begin{array}{l}\text { Sexual behavior with same-sex } \\
\text { partner }\end{array}$ & $236(59.0)$ & $191(63.7)$ & $45(45.0)$ & \\
\hline $\begin{array}{l}\text { Sexual behavior with } \\
\text { opposite-sex partner }\end{array}$ & $89(22.2)$ & 59 (19.7) & $30(30.0)$ & \\
\hline Other routes ${ }^{*}$ & $21(5.2)$ & $14(4.6)$ & $7(7.0)$ & \\
\hline Not sure & $54(13.6)$ & $36(12.0)$ & $18(18.0)$ & \\
\hline ART regimen & & & & 0.431 \\
\hline $\begin{array}{l}\text { Tenofovir, lamivudine, and } \\
\text { efavirenz }\end{array}$ & $336(84.0)$ & $255(85.0)$ & $81(81.0)$ & \\
\hline Other & $64(16.0)$ & $45(15.0)$ & $19(19.0)$ & \\
\hline $\begin{array}{l}\text { Psychosocial status ( ) } \\
\text { Social support (range 1-7) }\end{array}$ & & & & \\
\hline
\end{tabular}




\begin{tabular}{|c|c|c|c|c|}
\hline \multirow[t]{2}{*}{ Variables } & $\begin{array}{c}\text { All } \\
\text { participants } \\
(n=400)\end{array}$ & $\begin{array}{l}\text { Participants who } \\
\text { completed follow- } \\
\text { up surveys } \\
(n=300)\end{array}$ & $\begin{array}{c}\text { Participants who did } \\
\text { not complete follow- } \\
\text { up surveys } \\
(n=100)\end{array}$ & \multirow[t]{2}{*}{$P$} \\
\hline & $n(\%)$ & $n(\%)$ & $n(\%)$ & \\
\hline Family & $\begin{array}{l}4.80 \pm \\
1.45\end{array}$ & $4.79 \pm 1.45$ & $4.83 \pm 1.45$ & 0.807 \\
\hline Friends & $\begin{array}{l}4.86 \pm \\
1.37\end{array}$ & $4.90 \pm 1.35$ & $4.74 \pm 1.42$ & 0.316 \\
\hline Significant others & $\begin{array}{l}5.29 \pm \\
1.22\end{array}$ & $5.34 \pm 1.19$ & $5.13 \pm 1.28$ & 0.129 \\
\hline Social stigma (range 13-52) & $\begin{array}{l}29.80 \pm \\
6.77\end{array}$ & $29.86 \pm 6.68$ & $29.65 \pm 7.04$ & 0.792 \\
\hline \multicolumn{5}{|l|}{$\begin{array}{l}\text { Mental health problems }\left[\mathrm{M}\left(\mathrm{Q}_{1} \text {, }\right.\right. \\
\left.\left.\mathrm{Q}_{2}\right)\right]\end{array}$} \\
\hline Probable depression (range $0-27$ ) & $\begin{array}{l}7.00(4.00 \\
10.00)\end{array}$ & $7.00(4.00,9.00)$ & $7.00(3.00,10.00)$ & 0.504 \\
\hline $\begin{array}{l}\text { No (PHQ-9 score }<10 \text { ) } \\
\text { Yes (PHQ-9 score } \geq 10 \text { ) }\end{array}$ & $\begin{array}{l}296(74.0) \\
104(26.0)\end{array}$ & $\begin{array}{c}231(77.0) \\
69(23.0)\end{array}$ & $\begin{array}{l}65(65.0) \\
35(35.0)\end{array}$ & 0.025 \\
\hline $\begin{array}{l}\text { Moderate/severe anxiety (range } \\
0-27 \text { ) }\end{array}$ & $\begin{array}{l}6.00(2.00 \\
7.00)\end{array}$ & $6.00(2.00,7.00)$ & $4.00(2.00,8.00)$ & 0.833 \\
\hline $\begin{array}{l}\text { No (GAD-7 score }<10) \\
\text { Yes (GAD-7 score } \geq 10 \text { ) }\end{array}$ & $\begin{array}{l}340(85.0) \\
60(15.0)\end{array}$ & $\begin{array}{l}256(85.3) \\
44(14.7)\end{array}$ & $\begin{array}{l}84(84.0) \\
16(16.0)\end{array}$ & 0.872 \\
\hline
\end{tabular}

venous drug use, mother-to-child transmission, blood transfusion, occupational exposure.

Q2): Median (lower quartile, upper quartile)

\section{Changes in mental health problems from baseline to Month 12}

Among participants who completed all three surveys $(n=300)$, the prevalence of probable depression declined from $23.0 \%$ at baseline to $14.0 \%$ at Month 12 (paired $c^{2}=9.80, P=0.002$ ). The prevalence of moderate/severe anxiety was $14.7 \%$ at baseline and $8.7 \%$ at Month 12 (paired $c^{2}=5.16, P=0.023$ ). The depressive scores declined from $7.00(4.00,9.00)\left[M\left(Q_{1}, Q_{2}\right)\right]$ at baseline to $5.00(1.00,8.00)\left[M\left(Q_{1}, Q_{2}\right)\right]$ at Month $12(P<0.001)$. The anxious scores were $6.00(2.00,7.00)\left[M\left(Q_{1}, Q_{2}\right)\right]$ at baseline and $3.00(0.00,7.00)\left[M\left(Q_{1}, Q_{2}\right)\right]$ at Month 12 $(P<0.001)$.

\section{Experiences related to ART measured at Month 6}

Among participants who completed all three surveys $(n=300)$, most reported a rise in CD4 cell count $(90.0 \%)$, improvement in physical health status $(80.3 \%)$, and regained self-confidence $(74.7 \%)$. Some also reported improvement in relationships with sexual partners since ART initiation (21.7\%). On the negative side, $76.3 \%$ experienced inconvenience in daily life due to ART use and $50.7 \%$ suffered side effects of ART. Some participants also reported AIDS-related symptoms (18.3\%) and exposure of their HIV-positive status due to ART use $(22.7 \%)$.

\section{Associated factors of probable depression and moderate/severe anxiety measured at Month 12}

Participants who were female, received less support from friends and significant others, perceived greater social stigma, and had probable depression and moderate/severe anxiety at baseline were more likely than others to have probable depression at Month 12. Participants who were younger, had lower monthly personal income, received less support from friends, and perceived greater social stigma at baseline were more likely to have 
moderate/severe anxiety at Month 12 (Table 2). Stepwise regression models showed that sex, support from family and significant others, and probable depression at baseline affected depression at Month 12 independently. Support from friends and social stigma were independent factors associated with moderate/severe anxiety at Month 12 .

2 Associations between baseline variables and mental health problems at Month 12 (among those wh eted all three surveys: baseline, Month 6, and Month 12, $\mathrm{n}=300$ ) 


\begin{tabular}{|c|c|c|c|c|}
\hline \multirow[t]{2}{*}{ Variables } & \multicolumn{2}{|c|}{ Probable depression } & \multicolumn{2}{|c|}{ Moderate/Severe anxiety } \\
\hline & Row\% & $\mathrm{OR}_{\mathrm{u}}(95 \% \mathrm{CI})$ & Row\% & $\mathrm{OR}_{\mathrm{u}}(95 \% \mathrm{CI})$ \\
\hline \multicolumn{5}{|l|}{$\begin{array}{l}\text { Background characteristics } \\
\text { Age (vears) }\end{array}$} \\
\hline $18-$ & 16.9 & 1.00 & 13.8 & 1.00 \\
\hline 25- & 9.8 & $0.53(0.22,1.28)$ & 5.3 & $0.35(0.12,0.97)^{*}$ \\
\hline $35-$ & 17.1 & $1.02(0.41,2.53)$ & 11.4 & $0.80(0.28,2.24)$ \\
\hline $45-$ & 18.8 & $1.13(0.36,3.33)$ & 6.2 & $0.41(0.06,1.74)$ \\
\hline \multicolumn{5}{|l|}{ Sex } \\
\hline Female & 36.8 & 1.00 & 21.1 & 1.00 \\
\hline Male & 12.5 & $\begin{array}{l}0.24 \\
0.69)^{* *}\end{array}$ & 7.8 & $\begin{array}{l}0.32 \\
1.19)^{\dagger}\end{array}$ \\
\hline \multicolumn{5}{|l|}{ Education } \\
\hline Primary school and below & 16.5 & 1.00 & 8.8 & 1.00 \\
\hline Junior or senior high & 14.6 & $0.87(0.37,1.98)$ & 13.4 & $1.61(0.62,4.36)$ \\
\hline College and above & 11.8 & $0.68(0.31,1.48)$ & 5.5 & $0.61(0.20,1.75)$ \\
\hline \multicolumn{5}{|l|}{ Current marital status } \\
\hline Married or cohabiting with a partner & 14.4 & 1.00 & 10.6 & 1.00 \\
\hline Single & 14.4 & $1.00(0.50,2.04)$ & 7.8 & $0.71(0.31,1.69)$ \\
\hline Other & 10.3 & $0.68(0.15,2.28)$ & 6.9 & $0.63(0.09,2.52)$ \\
\hline \multicolumn{5}{|l|}{ Monthly personal income in RMB (USD) } \\
\hline No fixed income & 18.0 & 1.00 & 14.0 & 1.00 \\
\hline$<3000(<433$ USD $)$ & 17.1 & $0.94(0.37,2.50)$ & 12.9 & $0.91(0.31,2.71)$ \\
\hline $3000-(433-U S D)$ & 14.8 & $0.79(0.33,2.01)$ & 7.4 & $0.49(0.17,1.48)$ \\
\hline 5000- (722- USD) & 6.9 & $\begin{array}{l}0.34 \\
1.05)^{\dagger} \quad(0.10\end{array}$ & 2.8 & $0.18(0.03,0.77)^{*}$ \\
\hline \multicolumn{5}{|l|}{ Employment status } \\
\hline Full-time & 13.0 & 1.00 & 7.9 & 1.00 \\
\hline Part-time/unemployed/retired/student & 15.4 & $1.22(0.63,2.36)$ & 9.8 & $1.26(0.55,2.83)$ \\
\hline \multicolumn{5}{|l|}{ City of permanent residence } \\
\hline Guangzhou & 8.7 & 1.00 & 5.8 & 1.00 \\
\hline Other cities & 15.6 & $1.94(0.83,5.3)$ & 9.5 & $1.71(0.63,6.01)$ \\
\hline \multicolumn{5}{|l|}{ HIV/AIDS-related characteristics } \\
\hline \multicolumn{5}{|l|}{ Presence of AIDS-related symptoms } \\
\hline No & 14.8 & 1.00 & 9.5 & 1.00 \\
\hline Yes & 12.6 & $0.83(0.41,1.64)$ & 7.2 & $0.74(0.29,1.71)$ \\
\hline \multicolumn{5}{|l|}{ CD4 cell count (cell $\left./ \mathrm{mm}^{3}\right)$} \\
\hline$<200$ & 12.8 & 1.00 & 10.3 & 1.00 \\
\hline $200-$ & 15.6 & $1.25(0.57,2.92)$ & 8.9 & $0.85(0.34,2.27)$ \\
\hline $350-$ & 10.2 & $0.77(0.25,2.21)$ & 6.8 & $0.64(0.16,2.13)$ \\
\hline $500-$ & 17.9 & $1.48(0.42,4.63)$ & 7.1 & $0.67(0.10,2.90)$ \\
\hline \multicolumn{5}{|l|}{ Route of HIV transmission } \\
\hline Sexual behavior with same-sex partner & 12.0 & 1.00 & 8.4 & 1.00 \\
\hline $\begin{array}{l}\text { Sexual behavior with opposite-sex } \\
\text { partner }\end{array}$ & 15.3 & $1.31(0.55,2.94)$ & 6.8 & $0.80(0.22,2.28)$ \\
\hline Other routes* & 14.3 & $1.22(0.18,4.84)$ & 7.1 & $0.84(0.04,4.66)$ \\
\hline Not sure & 22.2 & $2.09(0.81,4.98)$ & 13.9 & $1.76(0.55,4.88)$ \\
\hline \multicolumn{5}{|l|}{ ART regimen } \\
\hline Tenofovir, lamivudine, and efavirenz & 13.7 & 1.00 & 8.2 & 1.00 \\
\hline Other & 15.6 & $1.16(0.45,2.66)$ & 11.1 & $1.39(0.45,3.65)$ \\
\hline \multicolumn{5}{|l|}{ Psychosocial status } \\
\hline \multicolumn{5}{|l|}{ Social support ( ) (range 1-7) } \\
\hline Family & N.A. & $\begin{array}{l}0.98 \\
1.24)\end{array}$ & N.A. & $0.83(0.64,1.09)$ \\
\hline Friends & N.A. & $\begin{array}{l}0.71 \\
0.90)^{* *}\end{array}$ & N.A. & $\begin{array}{l}0.61 \\
0.81)^{* * *}\end{array}$ \\
\hline Significant others & N.A. & $\begin{array}{l}0.71 \\
0.91)^{* *}\end{array}$ & N.A. & $\begin{array}{l}0.76 \\
1.03)^{\dagger}\end{array}$ \\
\hline
\end{tabular}




\begin{tabular}{|c|c|c|c|c|c|c|}
\hline \multirow{2}{*}{ Variables } & \multicolumn{3}{|c|}{ Probable depression } & \multicolumn{3}{|c|}{ Moderate/Severe anxiety } \\
\hline & Row\% & $\mathrm{OR}_{\mathrm{u}}\left(95^{\circ}\right.$ & & Row\% & $\mathrm{OR}_{\mathrm{u}}(95$ & \\
\hline Social stigma ( ) (range 13-52) & N.A. & $\begin{array}{l}1.06 \\
1.12)^{*}\end{array}$ & $(1.01$, & N.A. & $\begin{array}{l}1.08 \\
1.15)^{* *}\end{array}$ & $(1.02$, \\
\hline Mental health problems & & & & & & \\
\hline Probable depression (PHQ-9 score $\geq 10$ ) & 30.4 & $\begin{array}{l}4.37 \\
8.69)^{* * *}\end{array}$ & $(2.21$, & 14.5 & $\begin{array}{l}2.28 \\
5.22)^{\dagger}\end{array}$ & $(0.95$, \\
\hline $\begin{array}{l}\text { Moderate/severe anxiety (GAD-7 score } \\
\geq 10 \text { ) }\end{array}$ & 25.0 & $\begin{array}{l}2.42 \\
5.17)^{*}\end{array}$ & (1.07, & 15.9 & $\begin{array}{l}2.36 \\
5.79)^{\dagger}\end{array}$ & $(0.87$, \\
\hline
\end{tabular}

$<P<0.1 ;{ }^{*} P<0.05 ;{ }^{* *} P<0.01 ;{ }^{* * *} P<0.001$

ravenous drug use, mother-to-child transmission, blood transfusion, occupational exposure Not applicable.

After adjustment for baseline variables with $P<0.1$ in the univariate analysis plus baseline depression and anxiety status, those who reported improved physical health at Month 6 were less likely to have moderate/severe anxiety $\left(\mathrm{OR}_{\mathrm{a}}=0.31,95 \% \mathrm{Cl}: 0.12\right.$ to 0.84$)$ at Month 12 . Those reporting improved relationships with sexual partners at Month 6 were less likely to have either probable depression $\left(\mathrm{OR}_{\mathrm{a}}=0.25,95 \% \mathrm{Cl}: 0.06\right.$ to $0.73)$ or moderate/severe anxiety at Month $12\left(\mathrm{OR}_{\mathrm{a}}=0.17,95 \% \mathrm{Cl}: 0.02\right.$ to 0.71$)$, and those reporting regained self-confidence were also less likely to have probable depression $\left(\mathrm{OR}_{\mathrm{a}}=0.40,95 \% \mathrm{Cl}: 0.19\right.$ to 0.87$)$. AIDS-related symptoms were associated with probable depression $\left(\mathrm{OR}_{\mathrm{a}}=3.15,95 \% \mathrm{Cl}: 1.30\right.$ to 7.57$)$ at Month 12 . Side effects of ART and inconvenience in daily life due to ART use were associated with moderate/severe anxiety at Month 12 (Table 3).

Associations between ART experiences at Month 6 and mental health problems at Month 12 (among tho completed all three surveys: baseline, Month 6, and Month 12, $\mathrm{n}=300$ )

\begin{tabular}{|c|c|c|c|c|c|c|c|c|}
\hline \multirow{3}{*}{$\begin{array}{l}\text { Experiences during ART } \\
\text { Positive experiences } \\
\text { Increased CD4 cell count }\end{array}$} & \multicolumn{4}{|c|}{ Probable depression } & \multicolumn{4}{|c|}{ Moderate/severe anxiety } \\
\hline & \multicolumn{2}{|c|}{$\mathrm{OR}_{\mathrm{u}}(95 \% \mathrm{CI})$} & \multicolumn{2}{|c|}{$\begin{array}{l}\mathrm{OR}_{\mathrm{a}}{ }^{1}(95 \% \\
\mathrm{CI})\end{array}$} & \multicolumn{2}{|c|}{$\mathrm{OR}_{\mathrm{u}}(95 \% \mathrm{CI})$} & \multicolumn{2}{|c|}{$\mathrm{OR}_{\mathrm{a}}^{2}(95 \% \mathrm{CI})$} \\
\hline & & $(0.50$, & -- & & & (0.27, & -- & \\
\hline Improved physical health & $\begin{array}{l}0.42 \\
0.88)^{*}\end{array}$ & 1, & $\begin{array}{l}0.63 \\
1.47)\end{array}$ & $(0.28$ & $\begin{array}{l}0.29 \\
0.68)^{* *}\end{array}$ & $(0.13$ & $\begin{array}{l}0.31 \\
0.84)^{*}\end{array}$ & $(0.12$, \\
\hline $\begin{array}{l}\text { Improved relationships with sexual } \\
\text { partners }\end{array}$ & $\begin{array}{l}0.34 \\
0.89)^{*}\end{array}$ & $(0.10$, & $\begin{array}{l}0.25 \\
0.73)^{*}\end{array}$ & $(0.06-$ & $\begin{array}{l}0.28 \\
0.98)^{\dagger}\end{array}$ & $(0.04$, & $\begin{array}{l}0.17 \\
0.71)^{*}\end{array}$ & $(0.02$, \\
\hline Regained self-confidence & $\begin{array}{l}0.30 \\
0.60)^{* * *}\end{array}$ & $(0.16$, & $\begin{array}{l}0.40 \\
0.87)^{*}\end{array}$ & $(0.19-$ & $\begin{array}{l}0.36 \\
0.82)^{*}\end{array}$ & $(0.16$, & $\begin{array}{l}0.47 \\
1.21)\end{array}$ & $(0.19$, \\
\hline $\begin{array}{l}\text { Negative experience } \\
\text { Side effects of ART }\end{array}$ & & $(1$ & 2.07 & 9- & 3.59 & 8, & & .35 \\
\hline AIDS-related sy & $\begin{array}{l}2.00 \\
4.14)^{\dagger}\end{array}$ & 2, & $\begin{array}{l}3.15 \\
7.57)^{*}\end{array}$ & $*$ & 2.15 & 4, & 2.82 & .96, \\
\hline $\begin{array}{l}\text { Inconvenience in daily life due to } \\
\text { ART use }\end{array}$ & $\begin{array}{l}1.37 \\
3.33)\end{array}$ & .63 & - & & $\begin{array}{l}4.04 \\
25.54)^{\dagger}\end{array}$ & $(1.16$, & $\begin{array}{l}5.74 \\
41.92\end{array}$ & 1.36, \\
\hline $\begin{array}{l}\text { Exposure of HIV-positive status } \\
\text { due to ART use }\end{array}$ & $\begin{array}{l}0.65 \\
1.45)\end{array}$ & $(0.25$, & -- & & $\begin{array}{l}1.29 \\
3.08) \\
\end{array}$ & $(0.48$ & -- & \\
\hline
\end{tabular}

$<P<0.1 ;{ }^{*} P<0.05 ;{ }^{* *} P<0.01 ;{ }^{* * *} P<0.001$

significant in univariate analysis 
: Adjusted odds ratios, adjustment for mental health status measured at baseline (probable sion and moderate/severe anxiety) and baseline background variables with $P<0.1$ (sex, monthly ıl income, friend support, significant other support, and social stigma).

: Adjusted odds ratios, adjustment for mental health status measured at baseline (probable sion and moderate/severe anxiety) and baseline background variables with $P<0.1$ (age group, sex, y personal income, friend support, significant other support, and social stigma).

\section{Discussion}

Our research is one of the few longitudinal studies to investigate the association between ART-related experiences and mental health problems among PLWH who have recently started ART. In our study, the prevalence of probable depression and moderate/severe anxiety both at baseline and at Month 12 was lower than that reported by meta-analyses targeting PLWH in China $[9,42]$. This discrepancy may be attributable to the broad range of measurements used in different studies and to differences in the populations studied (e.g., gender identity, age, region, treatment status) $[20,21,43]$. In studies using the same measurements as in our study, the prevalence of depression was 39.3\% among newly diagnosed PLWH [44], and the prevalence of anxiety was $64.3 \%$ among those who had newly initiated ART [45]. Similar to previous longitudinal studies [22, 46], our study showed a significant decline in the prevalence of mental health problems at Month 12 compared to pre-treatment. Among those who remained in the cohort, baseline mental health status was a significant factor of mental health problems one year after starting ART. Screening for mental health problems and related care is greatly warranted for PLWH who were new to ART.

This study provides healthcare workers with insights for promoting mental health among PLWH who are starting ART, especially regarding segmentation. According to social marketing approaches, careful segmentation improves the effectiveness of health promotion programs [47]. Our study showed the characteristics of those who were more likely to drop out from the study cohort. As a significant of these participants also dropped out from ART, it indicated the need of more proactive intervention to reduce loss to follow-up among those who were older, had a lower or unfixed monthly income, had probable depression, and were infected through heterosexual behaviours and intravenous drug use at the beginning of ART program. Similar to previous studies, females are found to be at higher risk of mental health problems after receiving ART $[48,49]$. As female PLWH may suffer higher levels of internalized stigma and experience more social stigma than male patients [48, 49], more attention should be focused on female PLWH in future mental health promotion programs. Furthermore, PLWH without fixed incomes or with relatively low incomes should be prioritized in future programs, as they not only tend to drop out of programs but also to have a higher prevalence of mental health problems one year after receiving ART. Although ART is provided for free to all PLWH in China [50], PLWH still need to pay for compulsory physical examinations every three months. This financial burden may become a stressor, especially for those with lower incomes.

Similar to the findings of cross-sectional studies [10,51], we found that stronger social support and lower perceived social stigma at baseline were protective factors for mental health problems. A study among Chinese PLWH also showed that family and social networks, and the trust/intensity of relationships with family members and others played important roles in mental wellbeing [52]. In addition, studies have shown that living in a trusting social environment with lower stigma toward PLWH alleviates daily stressors and protects against 
mental health problems [52-54]. On the one hand, strategies to encourage and teach PLWH to communicate with their family/friends for support are needed [55]. On the other hand, public health education programs aimed at reducing discrimination against PLWH may help provide PLWH on ART with more social support to promote their mental health.

Our findings highlight the influence of ART-related experiences on mental health problems among PLWH, as a number of positive/negative experiences were significantly or marginally significantly associated with probable depression and/or moderate/severe anxiety after controlling for potential baseline confounders. Consistent with previous findings, side effects of ART may trigger depressive and anxiety symptoms $[20,21,27]$. About half of our participants experienced some side effects at Month 6 . These side effects bring physical discomfort to PLWH on ART, interrupting their normal daily and social functioning and reducing quality of life [31]. Thus, the development of effective interventions to facilitate self-management of side effects is greatly needed.

Participants with AIDS-related symptoms were more likely to develop probable depression, in line with previous studies [26, 27]. A review has reported a significant association between depressive symptoms and HIV progression [56]. The significant association may be due to the chronic impact of HIV on immune and diseaserelated parameters, which in turn may lead to depressive symptoms among PLWH on ART [57]. We found that about three quarters of the participants reported inconvenience in daily life due to ART use, and this was associated factor of moderate/severe anxiety. Taking ART every day may be a reminder of their HIV status and make them feel stressed [32]. Scaling up the single tablet regimen and reducing dose frequency could thus help to improve mental health among PLWH on ART.

Relatively few participants reported an improvement in their sex-partner relationship, but for those who did, it was protective against depressive and anxiety symptoms. Health communication messages should therefore be disseminated to PLWH and their sexual partners emphasizing that viral suppression by ART greatly reduces the risk of transmitting HIV through sex [1,2]. This may remove concerns about resuming sexual behaviour and improve intimacy between sexual partners. Most participants regained self-confidence during ART, and this was also protective against depressive symptoms. Studies have shown that ART makes people feel ready to return to normal life [31]. Our findings demonstrate the clinical benefits of the new "treat all" policy, as more than $80 \%$ of PLWH experienced improvements in physical health, which contributed to improved mental health, and nearly $90 \%$ experienced a rise in CD4 cell count.

However, our study has some limitations. First, as participants were recruited by non-probabilistic sampling, the findings may not be generalizable to all PLWH in China. Second, due to attrition bias, the prevalence of mental health problems at Month 12 may be underestimated, as those who did not complete both follow-up surveys might have worse psychosocial statuses. Selection bias may exist, as we could not obtain the characteristics of those who refused to join the study. Third, the positive and negative experiences related to ART were selfdeveloped and had not been validated. Further studies are encouraged to focus on ART-related experience exhaustively. Fourth, it's not clear whether the association between ART experiences and mood symptoms persists in a long term, as all the participants were new to ART in current study. Fifth, substance use may play a role in mood outcomes but it was not examined in the study. Moreover, the results were self-reported and thus subject to social desirability bias.

\section{Conclusions}

Page 13/18 
In conclusion, our study verified the benefit of ART on mental health status of PLWH and highlighted the role of ART-related experiences in mental health status improvement. Tailored interventions targeting specific experiences such as to reduce side effects, inconvenience in daily life due to treatment, and to enhance partner relationship are warranted in future.

\section{Abbreviations}

ART: antiretroviral therapy; PLWH: people living with HIV; UNAIDS: the Joint United Nations Programme on HIV/AIDS; PHQ-9: the Patient Health Questionnaire; GAD-7: the Generalized Anxiety Disorder Scale; MSPSS: the Multidimensional Scale of Perceived Social Support; CSSSs: the Chinese Courtesy Stigma Scales

\section{Declarations}

\section{Ethics approval and consent to participate}

Ethical approval for the study was obtained from the Institutional Review Board (IRB) of the School of Public Health, Sun Yat-sen University, Guangzhou, China (No: 2016-003). Informed consent was obtained from all individual participants in the study.

\section{Consent for publication}

Not applicable.

\section{Availability of data and materials}

The datasets used and/or analysed during the current study are available from the corresponding author on reasonable request.

\section{Competing interests}

The authors declare that they have no competing interests.

\section{Funding}

This research was funded by the National Natural Science Foundation of China (71774178), Science and Technology Planning Project of Guangdong (2017A020212006), the Guangzhou Science and Technology Project (201607010368), and the National Science and Technology Major Project of the Ministry of Science and Technology of China (2018ZX10715004).

\section{Authors' contributions}

JX and ZW contributed equally to this study. JG, ZW, and JX designed the study.

QL, QH, GX, YL, and KZ performed data collection; JX, ZW, and QH conducted statistical analyses of the data. JX and ZW drafted the paper. LL and JG provided comments to the draft paper. All authors read and approved the final manuscript. 
The authors would like to thank all health workers of the Department of Infectious Diseases, Guangzhou Eighth People's Hospital. We extend thanks to all participants of the study.

\section{References}

1. Baeten JM, Donnell D, Ndase P, Mugo NR, Campbell JD, Wangisi J, et al. Antiretroviral prophylaxis for HIV prevention in heterosexual men and women. The New England journal of medicine. 2012;367(5):399-410.

2. When To Start C, Sterne JA, May M, Costagliola D, de Wolf F, Phillips AN, et al. Timing of initiation of antiretroviral therapy in AIDS-free HIV-1-infected patients: a collaborative analysis of 18 HIV cohort studies. Lancet. 2009;373(9672):1352-1363.

3. UNAIDS. 90-90-90 An ambitious treatment target to help end the AIDS epidemic; 2014. http://www.unaids.org/sites/default/files/media_asset/90-90-90_en_0.pdf. Accessed 17 Sep 2020.

4. UNAIDS. Country progress report - China; 2018.

https://www.unaids.org/sites/default/files/country/documents/CHN_2018_countryreport.pdf. Accessed 17 Sep 2020.

5. WHO. Guideline on when to start antiretroviral therapy and on pre-exposure prophylaxis for HIV; 2015. https://apps.who.int/iris/bitstream/handle/10665/186275/9789241509565_eng.pdf. Accessed 17 Sep 2020.

6. Taylor BS, Tieu HV, Jones J, Wilkin TJ. CROI 2019: advances in antiretroviral therapy. Top Antivir Med. 2019;27(1):50-68.

7. Frank TD, Carter A, Jahagirdar D, Biehl MH, Douwes-Schultz D, Larson SL, et al. Global, regional, and national incidence, prevalence, and mortality of HIV, 1980-2017, and forecasts to 2030, for 195 countries and territories: a systematic analysis for the Global Burden of Diseases, Injuries, and Risk Factors Study 2017. Lancet HIV. 2019;6(12):e831-e859.

8. Webster P. UNAIDS survey aligns with so-called fourth 90 for HIV/AIDS. The Lancet. 2019;393(10187):2188.

9. Niu L, Luo D, Liu Y, Silenzio VM, Xiao S. The Mental Health of People Living with HIV in China, 1998-2014: A Systematic Review. PLoS One. 2016;11(4):e0153489.

10. Prati G, Zani B, Pietrantoni L, Scudiero D, Perone P, Cosmaro L, et al. PEP and TasP Awareness among Italian MSM, PLWHA, and High-Risk Heterosexuals and Demographic, Behavioral, and Social Correlates. PLoS One. 2016;11(6):e0157339.

11. Luo R, Silenzio VMB, Huang Y, Chen X, Luo D. The Disparities in Mental Health between Gay and Bisexual Men Following Positive HIV Diagnosis in China: A One-Year Follow-Up Study. Int J Environ Res Public Health. 2020;17(10):3414.

12. Rogers BG, Lee JS, Bainter SA, Bedoya CA, Pinkston M, Safren SA. A multilevel examination of sleep, depression, and quality of life in people living with HIV/AIDS. J Health Psychol. 2020;25(10-11):1556-1566.

13. Catalan J, Tuffrey V, Ridge D, Rosenfeld D, Team $H$. What influences quality of life in older people living with HIV? AIDS Res Ther. 2017;14:22.

14. McMahon JM, Braksmajer A, Zhang C, Leblanc N, Chen M, Aidala A, et al. Syndemic factors associated with adherence to antiretroviral therapy among HIV-positive adult heterosexual men. AIDS Res Ther. 
2019;16(1):32.

15. Nachega JB, Mutamba B, Basangwa D, Nguyen H, Dowdy DW, Mills EJ, et al. Severe mental illness at ART initiation is associated with worse retention in care among HIV-infected Ugandan adults. Trop Med Int Health. 2013;18(1):53-57.

16. Saag LA, Tamhane AR, Batey DS, Mugavero MJ, Eaton EF. Mental health service utilization is associated with retention in care among persons living with HIV at a university-affiliated HIV clinic. AIDS Res Ther. 2018;15(1):1.

17. Mayston R, Kinyanda E, Chishinga N, Prince M, Patel V. Mental disorder and the outcome of HIV/AIDS in low-income and middle-income countries: a systematic review. AIDS. 2012;26 Suppl 2:117-135.

18. Tran BX, Dang AK, Truong NT, Ha GH, Nguyen HLT, Do HN, et al. Depression and Quality of Life among Patients Living with HIV/AIDS in the Era of Universal Treatment Access in Vietnam. Int J Environ Res Public Health. 2018;15(12):2888.

19. Lowther K, Selman L, Harding R, Higginson IJ. Experience of persistent psychological symptoms and perceived stigma among people with HIV on antiretroviral therapy (ART): a systematic review. Int J Nurs Stud. 2014;51(8):1171-1189.

20. Rong H, Nianhua X, Jun X, Lianguo R, Si W, Sheng W, et al. Prevalence of and risk factors for depressive symptoms among people living with HIV/AIDS receiving antiretroviral treatment in Wuhan, China: a short report. AIDS Care. 2017;29(12):1524-1528.

21. Liu H, Zhao M, Ren J, Qi X, Sun H, Qu L, et al. Identifying factors associated with depression among men living with HIV/AIDS and undergoing antiretroviral therapy: a cross-sectional study in Heilongjiang, China. Health Qual Life Outcomes. 2018;16(1):190.

22. Eaton EF, Gravett RM, Tamhane AR, Mugavero MJ. Antiretroviral Therapy Initiation and Changes in SelfReported Depression. Clin Infect Dis. 2017;64(12):1791-1794.

23. Martinez P, Tsai AC, Muzoora C, Kembabazi A, Weiser SD, Huang Y, et al. Reversal of the Kynurenine pathway of tryptophan catabolism may improve depression in ART-treated HIV-infected Ugandans. J Acquir Immune Defic Syndr. 2014;65(4):456-462.

24. Manne-Goehler J, Montana L, Gomez-Olive FX, Rohr J, Harling G, Wagner RG, et al. The ART Advantage: Health Care Utilization for Diabetes and Hypertension in Rural South Africa. J Acquir Immune Defic Syndr. 2017;75(5):561-567.

25. Tesfaw G, Ayano G, Awoke T, Assefa D, Birhanu Z, Miheretie G, et al. Prevalence and correlates of depression and anxiety among patients with HIV on-follow up at Alert Hospital, Addis Ababa, Ethiopia. BMC Psychiatry. 2016;16(1):368.

26. Asangbeh SL, Sobngwi JL, Ekali GL, Eyoum C, Msellati P. Predictors of depression among patients on art in a rural health district in North West Cameroon. AIDS Care. 2016;28(2):205-208.

27. Beyene Gebrezgiabher B, Huluf Abraha T, Hailu E, Siyum H, Mebrahtu G, Gidey B, et al. Depression among Adult HIV/AIDS Patients Attending ART Clinics at Aksum Town, Aksum, Ethiopia: A Cross-Sectional Study. Depress Res Treat. 2019;doi: 10.1155/2019/3250431.

28. Li TS, Tubiana R, Katlama C, Calvez V, Mohand HA, Autran B. Long-lasting recovery in CD4 T-cell function and viral-load reduction after highly active antiretroviral therapy in advanced HIV-1 disease. The Lancet. 1998;351(9117):1682-1686.

Page 16/18 
29. Gakhar H, Kamali A, Holodniy M. Health-related quality of life assessment after antiretroviral therapy: a review of the literature. Drugs. 2013;73(7):651-672.

30. Kim MJ, Kim SW, Chang HH, Kim Y, Jin S, Jung H, et al. Comparison of Antiretroviral Regimens: Adverse Effects and Tolerability Failure that Cause Regimen Switching. Infect Chemother. 2015;47(4):231-238.

31. Chen WT, Shiu CS, Yang JP, Simoni JM, Fredriksen-Goldsen KI, Lee TS, et al. Antiretroviral Therapy (ART) Side Effect Impacted on Quality of Life, and Depressive Symptomatology: A Mixed-Method Study. J AIDS Clin Res. 2013;4:218.

32. De Los Rios P, Young B, Marcotullio S, Punekar Y, Koteff J, Ustianowski A, et al. Experiences and Emotional Challenges of Antiretroviral Treatment (ART)-Findings from the Positive Perspectives Study. Open Forum of Infectious Diseases. 2019;6 Suppl 2:481.

33. Guangzhou Eighth People's Hospita. Introduction to the infectious clinic; 2019. http://www.gz8h.com.cn/info/1499/2601.htm. Accessed 17 Sep 2020.

34. Wang W, Bian Q, Zhao Y, Li X, Wang W, Du J, et al. Reliability and validity of the Chinese version of the Patient Health Questionnaire (PHQ-9) in the general population. Gen Hosp Psychiatry. 2014;36(5):539-544.

35. Chen $S$, Chiu $H, X u B, M a Y$, Jin $T$, Wu M, et al. Reliability and validity of the PHQ-9 for screening late-life depression in Chinese primary care. Int J Geriatr Psychiatry. 2010;25(11):1127-1133.

36. Edwards M, Quinlivan EB, Bess K, Gaynes BN, Heine A, Zinski A, et al. Implementation of PHQ-9 depression screening for HIV-infected patients in a real-world setting. J Assoc Nurses AIDS Care. 2014;25(3):243-252.

37. Tong X, An D, McGonigal A, Park SP, Zhou D. Validation of the Generalized Anxiety Disorder-7 (GAD-7) among Chinese people with epilepsy. Epilepsy Res. 2016;120:31-36.

38. Spitzer RL, Kroenke K, Williams JB, Lowe B. A brief measure for assessing generalized anxiety disorder: the GAD-7. Arch Intern Med. 2006;166(10):1092-1097.

39. Zimet GD, Dahlem NW, Zimet SG, Farley GK. The Multidimensional Scale of Perceived Social Support. J Per Assess. 1988;52(1):30-41.

40. Zhou KN, Lie HX, Wei XL, Yin J, Liang PF, Zhang HM, et al. Reliability and validity of the multidimensional scale of perceived social support in Chinese mainland patients with methadone maintenance treatment. Compr Psychiat. 2015;60:182-188.

41. Liu H, Xu Y, Sun Y, Dumenci L. Measuring HIV stigma at the family level: psychometric assessment of the Chinese Courtesy Stigma Scales (CCSSs). PLoS One. 2014;9(3):e92855.

42. Wang T, Fu H, Kaminga AC, Li Z, Guo G, Chen L, et al. Prevalence of depression or depressive symptoms among people living with HIV/AIDS in China: a systematic review and meta-analysis. BMC Psychiatry. 2018;18(1):160.

43. Tao J, Vermund SH, Lu H, Ruan Y, Shepherd BE, Kipp AM, et al. Impact of Depression and Anxiety on Initiation of Antiretroviral Therapy Among Men Who Have Sex with Men with Newly Diagnosed HIV Infections in China. AIDS Patient Care STDS. 2017;31(2):96-104.

44. Huang Y, Luo D, Chen X, Zhang D, Huang Z, Xiao S. Role of psychosocial status in predicting health-related quality of life at 1-year follow-up among newly diagnosed people living with HIV. PLoS One. 2019;14(10):e0224322.

45. Yu Y, Luo D, Chen X, Huang Z, Wang M, Xiao S. Medication adherence to antiretroviral therapy among newly treated people living with HIV. BMC Public Health. 2018;18(1):825. 
46. Manne-Goehler J, Kakuhikire B, Abaasabyoona S, Barnighausen TW, Okello S, Tsai AC, et al. Depressive Symptoms Before and After Antiretroviral Therapy Initiation Among Older-Aged Individuals in Rural Uganda. AIDS Behav. 2019;23(3):564-571.

47. Valente TW, Fosados R. Diffusion of innovations and network segmentation: the part played by people in promoting health. Sexually transmitted diseases. 2006;33 Suppl 7:23-31.

48. Krumme AA, Kaigamba F, Binagwaho A, Murray MB, Rich ML, Franke MF. Depression, adherence and attrition from care in HIV-infected adults receiving antiretroviral therapy. J Epidemiol Community Health. 2015;69(3):284-289.

49. Loutfy MR, Logie CH, Zhang Y, Blitz SL, Margolese SL, Tharao WE, et al. Gender and Ethnicity Differences in HIV-related Stigma Experienced by People Living with HIV in Ontario, Canada. PLoS One. 2012;7(12):e48168.

50. Yang X, Wang Z, Wang X, Ma T, Xue H, He Y, et al. Behavioral Intention to Initiate Antiretroviral Therapy (ART) Among Chinese HIV-Infected Men Who Have Sex With Men Having High CD4 Count in the Era of "Treatment for All". Am J Mens Health. 2019;13(1):1557988319828615.

51. Li J, Mo PK, Wu AM, Lau JT. Roles of Self-Stigma, Social Support, and Positive and Negative Affects as Determinants of Depressive Symptoms Among HIV Infected Men who have Sex with Men in China. AIDS Behav. 2017;21(1):261-273.

52. Han J, Jia P, Huang Y, Gao B, Yu B, Yang S, et al. Association between social capital and mental health among older people living with HIV: The Sichuan Older HIV-Infected Cohort Study (SOHICS). BMC Public Health. 2020;20(1):581.

53. Mburu G, Ram M, Skovdal M, Bitira D, Hodgson I, Mwai GW, et al. Resisting and challenging stigma in Uganda: the role of support groups of people living with HIV. J Int AIDS Soc. 2013;16 Suppl 2:18636.

54. Odek WO. Social networks and mental health among people living with human immunodeficiency virus (HIV) in Johannesburg, South Africa. AIDS Care. 2014;26(8):1042-1049.

55. Maman S, van Rooyen H, Groves AK. HIV status disclosure to families for social support in South Africa (NIMH Project Accept/HPTN 043). AIDS Care. 2014;26(2):226-232.

56. Rabkin JG. HIV and depression: 2008 review and update. Curr HIV/AIDS Rep. 2008;5(4):163-171.

57. Leserman J. Role of depression, stress, and trauma in HIV disease progression. Psychosom Med. 2008;70(5):539-545. 